\title{
Perinatology clinic in the coronavirus disease-2019 pandemic: what harms, often teaches
}

\author{
Elif Gul Yapar Eyi, Ozlem Moraloglu Tekin, Arda Buglagil, Dilek Sahin, Aykan \\ Yucel, Atakan Tanacan, Filiz Halici Ozturk, Betul Yakistiran, Didem Yucel \\ Yetiskin, Serpil Unlu \& Derya Gokcinar
}

To cite this article: Elif Gul Yapar Eyi, Ozlem Moraloglu Tekin, Arda Buglagil, Dilek Sahin, Aykan Yucel, Atakan Tanacan, Filiz Halici Ozturk, Betul Yakistiran, Didem Yucel Yetiskin, Serpil Unlu \& Derya Gokcinar (2021) Perinatology clinic in the coronavirus disease-2019 pandemic: what harms, often teaches, The Journal of Maternal-Fetal \& Neonatal Medicine, 34:21, 3591-3600, DOI: 10.1080/14767058.2021.1875440

To link to this article: https://doi.org/10.1080/14767058.2021.1875440

View supplementary material $\asymp$

Published online: 28 Feb 2021.

Submit your article to this journal $\pi$

Џ Article views: 396

Q View related articles $\widetilde{ }$

View Crossmark data $\nearrow$ 


\title{
Perinatology clinic in the coronavirus disease-2019 pandemic: what harms, often teaches
}

\author{
Elif Gul Yapar Eyi ${ }^{a}$, Ozlem Moraloglu Tekin ${ }^{a}$ (D) Arda Buglagil ${ }^{b}$, Dilek Sahin $^{a}$ (D) Aykan Yucel $^{a}$, \\ Atakan Tanacan ${ }^{\mathrm{a}}$, Filiz Halici Ozturk ${ }^{\mathrm{a}}$ (D), Betul Yakistiran ${ }^{\mathrm{a}}$, Didem Yucel Yetiskin ${ }^{\mathrm{a}}$, Serpil Unlu ${ }^{\mathrm{a}}$ and \\ Derya Gokcinar ${ }^{\mathrm{a}}$
}

\author{
${ }^{a}$ Ministry of Health Ankara City Hospital, Bilkent, Ankara, Turkey; ${ }^{b}$ Department of Electrical and Electronics Engineering, Bilkent \\ University, Bilkent, Ankara, Turkey
}

\begin{abstract}
Background: Compartmental models simplify the mathematical modeling of infectious diseases based on reported cases. In the absence of precautions, personal protective equipment, quarantine and social distancing, a Susceptible-Exposed-Infectious-Recovered (SuEIR) model with Unscented Kalman Filter for coronavirus disease-19 (COVID-19) Forecasts in Turkey has revealed 174641 infected people on August 15, 2020, whilst the reported case was 12216 . Through numerical experiments, the effects of quarantine, social distancing, and COVID-19 testing on the dynamics of the outbreak varies. We herein present the documentation of the work in a perinatology clinic during COVID-19 pandemic to find the reflection in a pandemic hospital as even in the pandemic, pregnancy complications and fetal diagnosis/therapy are time-sensitive and cannot be delayed. During the prevention of the horizontal transmission to the health-care workers (HCWs), testing all pregnant women with nasopharyngeal/oropharyngeal swabs for severe acute respiratory syndrome coronavirus (SARS-COV-2) undergoing birth, ultrasound examinations, invasive procedures appear to be the gold standard so that appropriate precautions can be taken if the screen is positive. Though it is logical, it may be incompatible with a busy obstetric practise as a pending polymerase chain reaction (PCR) result should never delay any emergent procedure.

Objective: We aim to describe the development of COVID-19 disease of $408 \mathrm{HCW}$ out of 1462 by the exposure to pregnant women while providing obstetric care in a single tertiary perinatology unit under strict clinical triage, recommended precautions and wearing personal protective equipment and compare the maternal and perinatal outcome with those of the preceding three months.

Study design: A prospective cohort study involving the pregnant women and the HCW with positive PCR for SARS-COV-2 were carried out to correlate with the horizontal transmission while documenting the perinatal work.

Results: $25 \mathrm{HCW}$, including nurses/midwives: 11, doctors: 7 and health technicians: 3 and support staff: 4 developed positive PCR for SARS-COV-2 while providing healthcare to 162 cases: mild-moderate $(n=146)$, severe $(n=12)$ and critical $(n=1)$ and asymptomatic $(n=3)$ in obstetric population. 22 out of $25 \mathrm{HCW}$ were working in the perinatology unit. COVID-19 clinic was asymptomatic $(n=8)$, mild-moderate $(13)$ or severe $(n=2)$ in HCW. However, "Exposed" group in the SuEIR model, both the pregnant women and the HCW that have already been infected and have not been tested, which have been also capable of infecting the "Susceptible" group could not be determined. Some of the $\mathrm{HCW}$ and the pregnant women in the "Exposed" group were tested and transferred to the "Infectious" group (which were reported to be PCR positive), while the rest of them who recovered, transitted to the so-called "Unreported Recovered" group. The ratio of the women with severe preeclampsia admitted to intensive care unit increased significantly during the lockdown $(p=.01)$.

Conclusions: In a nonstop pandemic perinatology clinic, exposure to 162 PCR positive pregnant women may be correlated with a 5.4\% (22/408) documented horizontal transmission in the frontline HCW despite clinical triage and personal protective equipment.
\end{abstract}

ARTICLE HISTORY Received 28 September 2020 Revised 2 December 2020 Accepted 10 January 2021

\section{KEYWORDS}

Coronavirus disease-19 in pregnancy; virus transmission; health-care workers; physical distancing; personal protective equipment

\section{Introduction}

Although coronavirus disease-2019 (COVID-19) pandemic has ravaged humanity, all pregnant women, including those with confirmed or suspected COVID-
19 , must be able to access the right care at the right time. In Turkey, 1183652 live births occurred in 2019 [1]. The high number of births, high-risk conditions and unpreventable abortions have mandated the 
continuation of obstetric care during the pandemic. Moreover, Turkey has an overall cesarean section (CS) rate of 53.1 percentage, with repeat CS contributing to the rate in $24.4 \%$ [2]. As the announcement of the COVID-19 outbreak as a pandemic by the World Health Organization on 11 March 2020, and the declaration of the emergence of the first COVID-19 case in Turkey, the workload on obstetrics, dealing with two patients (mother and fetus) and having the responsibility of both, $24 \mathrm{~h}$ a day, independent of holidays and weekends, has aggravated.

Although there has been a busy obstetric practice, COVID-19 pandemics has been modeled for Turkey based on reported cases with the (Susceptible-Exposed- Infectious-Recovered (SuEIR) model [3] with Unscented Kalman Filter [4] for COVID19 Forecasts (Supplementary Video 1), a variant of the compartmental model in epidemiology, considering both untested and unreported cases. The assumption of the model based on a transition from a virtual "Quarantined/Isolated" group to the "Susceptible" group at a certain rate after the end of the lock-down on 1 June 2020. The rates, along with other model parameters are jointly estimated based on the Ministry of Health reported data. More specifically, "Exposed" group in the SuEIR model imply the individuals that have already been infected and have not been tested, which are also capable of infecting the "Susceptible" group. Moreover, some of the people in the "Exposed" group can get tested and transferred to the "Infectious" group (which are reported to the public), while the rest of them will recover/die and transit to the so-called Unreported Recovered group (which are not reported to the public) $[3,4]$.

While uncertainty in many aspects of COVID-19 is going on, the obstetric health-care workers (HCW) have augmented risks for COVID-19 due to the nonstop obstetric care. Not only women with COVID-19, but also pre-symptomatic and asymptomatic people [5-10] may create those risks on exposed groups. Social distancing, clinical triage and personal protective equipment (PPE) are the current recommendations to prevent person to person (horizontal) transmission [11-14]. However, physical distancing could not be achieved during births and ultrasound evaluation as the distance between the ultrasonography operator and the pregnant woman is $60 \mathrm{~cm}$ or less.

We tabulate the procedures from a nonstop perinatology clinic under triage during COVID-19 pandemic to estimate horizontal transmission risk assessment for HCW.

\section{Materials and methods}

A prospective cohort study involving the perinatology clinic during COVID-19 pandemic was carried out to evaluate the clinical course of the pregnant women with COVID-19 admitted to one of the largest and the latest hospital in Europe and the world (Figure 1) and assess the risk estimates of the COVID-19 transmission for the HCW. A HCW is one who delivers care and services to the sick and ailing either directly as doctors, nurses/midwives, health technicians or indirectly as pharmacists or providers of support services: e.g. data processing employees or security employees. The study was approved by the Hospital Ethics committee and the Ministry of Health (E1-20-808) and the tenets of the Declaration of the Helsinki was followed. During the COVID-19 pandemic, 3660-bed capacity Hospital was re-organized. One of the units of the hospital is the Maternity hospital with an area of $105878 \mathrm{~m}$ squares. $408 \mathrm{HCW}$ out of 1462 are working in the perinatology clinic (Figures 1 and 2) Declaration of COVID19 pandemic informed consent agreement was taken from all pregnant women in the hospital. Prioritizing the triage of patients with respiratory symptoms, triage for COVID-19 included the published protocols of the Ministry of Health [12] and performed by the midwives/nurses:

- $\operatorname{Fever}\left(>38^{\circ} \mathrm{C}\right)$

- At least one sign or symptom of respiratory disease (e.g. cough or shortness of breath, sore throat, running nose)

- Travel or residence in another country where COVID-19 is spreading during the 14 days before symptom onset?

- Contact with an individual with suspected or confirmed COVID-19 during the 14 days prior to symptom onset?

If one of the triage criteria was positive for either the pregnant women or the HCW, as entrances were limited to the accompanying person, those were separated and placed in a special single-person room with the door closed in a special unit in Obstetrics and Gynecology Emergency Area. In this area, there is also a negative pressure operating theater. The infectious disease specialist was notified. Hospital infection control program was initiated. In this unit, all HCW caring for the patient adhered to standard, contact and droplet precautions. All pregnant women informed to wear facial masks. Minimum HCW with designated roles entered the room and wore appropriate personal protective equipment (respirator, surgical gown, goggle, 
N95, face protective shield) [11-14] and took care of the women in cases of suspected and confirmed cases. After informing the women, nasopharyngeal/oropharyngeal PCR samples were taken. Diagnosis depended on detection of SARS-CoV2 (Severe acute respiratory syndrome coronavirus 2) in samples evaluated by Real-Time Reverse Transcriptase Polymerase Chain Reaction (RT-PCR) method targeting (RNA dependent RNA polymerase (RdRp) gene $[15,16]$ with the previously defined methods. Cycle threshold $(\mathrm{Ct})$ values of less than 40 were defined as positive. [6]

COVID-19 cases were defined as:

- Asymptomatic: Women who tested positive for SARS-CoV-2 by PCR but had no symptoms

- Mild: Women who had any of the various signs and symptoms (e.g. fever, cough, sore throat, malaise, headache, muscle pain) without shortness of breath, dyspnea, or abnormal chest imaging

- Moderate: Women who had evidence of lower respiratory disease by clinical assessment or imaging and saturation of oxygen $\left(\mathrm{SpO}_{2}\right) \geq 94 \%$

- Severe: Women who had respiratory frequency $>30$ breaths per minute, $\mathrm{SpO}_{2}<94 \%$, ratio of the arterial partial pressure of oxygen to fraction of inspired oxygen $<300 \mathrm{mmHg}$ or lung infiltrates $>50 \%$
- Critical: Women who had respiratory failure, septic shock, and/or multiple organ dysfunction [17-18]

HCW wore surgical masks, goggles and face shields during antenatal care, ultrasonography examinations, births, postpartum care and used N95 for cesarean sections (CS) and procedures requiring close contact. In an attempt to evaluate the perinatology workload during the pandemic, the number of the pregnant women in outpatient, inpatient clinics and level II Perinatology Intensive Care Unit (ICU) was evaluated and compared with those in the preceding three months. Births, fetal invasive procedures, embryonic and fetal deaths, induced abortions, births, CS rates and early neonatal outcomes were tabulated. As a part of routine practice, HCW were educated to regularly monitor themselves for fever and symptoms of COVID-19. If HCW developed fever $(\geq 38 \mathrm{C})$, symptoms consistent with COVID-19, while at work, or contact or suspicion of contact, they were evaluated by the specialist in infectious disease. PCR samples were obtained by the consent of the specialists of infectious disease $^{9}$ according to the updated guidelines of the Ministry of Health. Statistical analyses were performed using the Statistical Package for the Social Sciences (SPSS.22, IBM SPSS Statistics for Windows, Version 22.0 Armonk, NY: IBM Corp.). Video was prepared by Arda

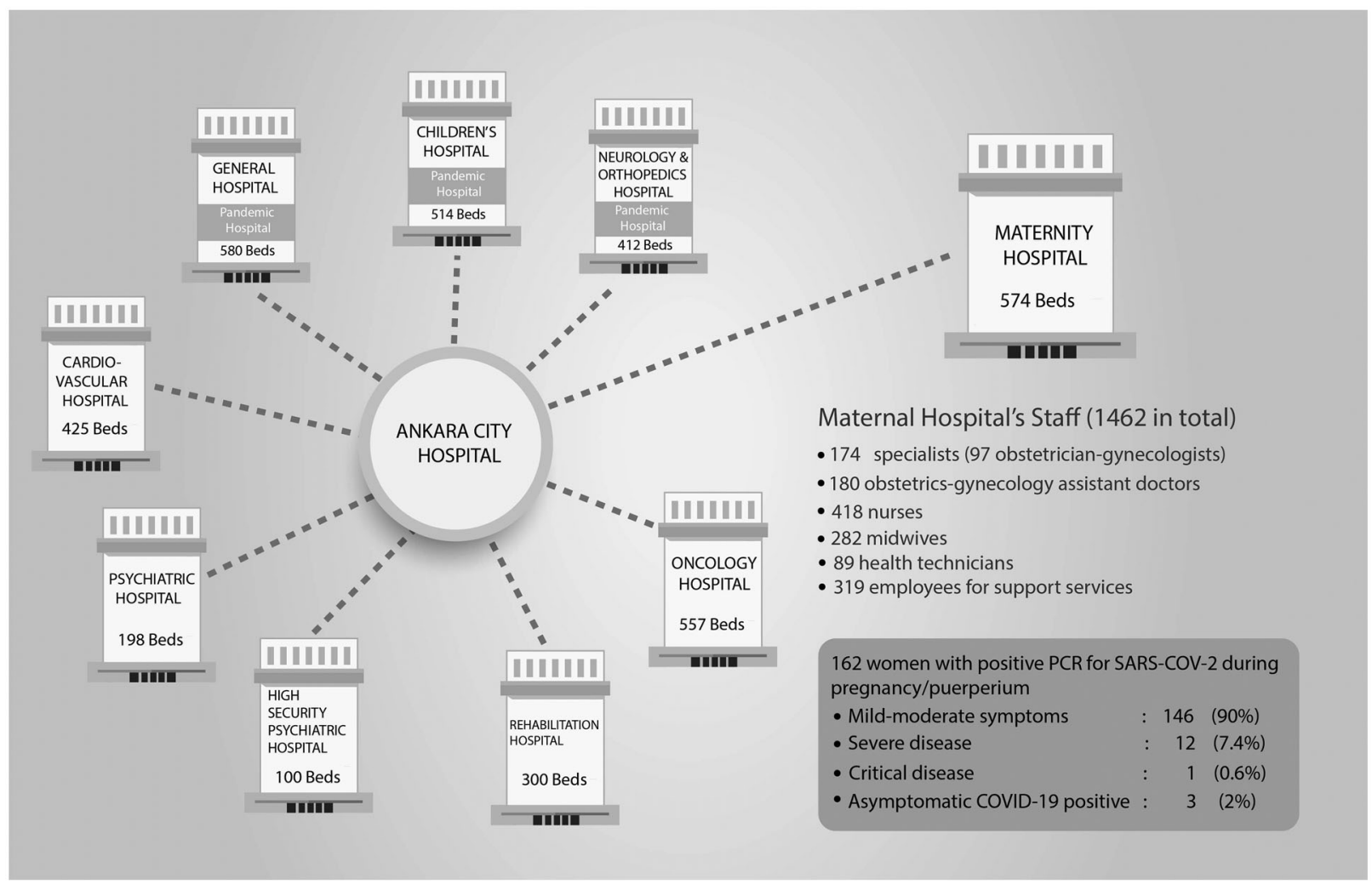

Figure 1. Hospital organization and distribution of the health-care workers. 
Buglagil using SuEIR model [3] in Kalman Filter [4]. Statistics involving pregnant women and HCW with COVID-19 are descriptive only. We used the chi-square test to compare categorical variables between the groups ( $p \leq .05$ was regarded as significant) and Adobe Illustrator CC 2019 for the figures.

\section{Results}

Between 1 December 2019 and 15 August 2020, Ankara City Hospital became the epicenter of the pandemic with 7425 COVID-19 patients confirmed by PCR. Of those, 99 died giving a fatality rate of $1.33 \%$. 83,485 tests were performed and 11,984 were positive. Among those, 162 (1.2\%) women having RT PCR positivity for SARS COV-2 during pregnancy and in the puerperium were in the follow up of the perinatology team. Of those while 29 RT PCR positivity for SARS COV-2 were detected up to 5 June, in the 31 July, there were 63 new cases. By the mid-August, a further 70 cases had occurred, making a sum of 162 .

$90.0 \%$ of the pregnant women with SARS COV-2 exhibited mild-moderate COVID-19 (Figure 1). Severe disease was present in $12(7.4 \%)$ and critical disease in one woman (0.6\%).Demographics, clinical severity, distribution of the gestational age, co-morbidities, duration of hospitalization and the pregnancy outcome of the women with SARS COV-2 PCR positivity were depicted in Tables 1 and 2. SARS-COV-2 positivity did occur at any time during pregnancy with co-morbidities and obstetric complications (Tables 1 and 2). Clinical scenarios are various. After an emergency tubal ectopic pregnancy rupture, PCR sampling revealed SARS-CoV-2 positivity. Another woman in whom second-trimester pregnancy was terminated due to critical aortic stenosis in the perinatology department and then referred to cardio-vascular surgery was found to be SARS-CoV-2 positive just before cardiac surgery. In addition to the respiratory symptoms in severe COVID-19, two of the women had complicated diabetes with diabetic ketoacidosis in whom perinatal death occurred at 26 weeks of gestation within $5 \mathrm{~h}$ after referral in one. Her diagnosis of diabetes was established at age 3 and she presented with Kussmaul breathing [19], respiratory compensation for metabolic acidosis, most commonly occurring in diabetic ketoacidosis. Her admission arterial blood gas evaluation during Kussmaul breathing showed a pH: 6.9 (normal levels: 7.37-7.45), $\mathrm{pCO}_{2}$ : 10.5 (normal levels: 35-46), - base excess: -27.9 (normal levels: 27.2-26.3), $\mathrm{pO}_{2:} 49.8$ (normal levels: 70-100) characterized by low partial pressure of carbon dioxide in conjunction with low bicarbonate because of forced
Table 1. Demographics of the pregnant women with COVID-19.

\begin{tabular}{lcc}
\hline & Median (IQR) & Range \\
\hline Age & $27(8)$ & $15-45$ \\
Parity & $1(1)$ & $0-6$ \\
BMI & $26.4(7)$ & $18-44$ \\
Duration of hospitalization(day) & $6 .(1)$ & $1-20$ \\
Co-morbidities & $n$ & $\%$ \\
Urinary tract infection & 12 & 7.4 \\
Anemia & 12 & 7.4 \\
Hypothyroidism & 9 & 5.5 \\
Obesity (BMI >30) & 9 & 5.5 \\
Hypertension & 2 & 1.2 \\
Heart disease & 3 & 1.8 \\
Type I diabetes + & & \\
diabetic ketoacidosis & 2 & 1.2 \\
Type II diabetes mellitus & 1 & 0.6 \\
Deep vein thrombosis in the leg & 2 & 1.2 \\
Thrombocytopenia & 2 & 1.2 \\
Chronic kidney disease & 1 & 0.6 \\
Asthma & 1 & 0.6 \\
Beta thalassemia & 1 & 0.6 \\
Epilepsy & 1 & 0.6 \\
Ovarian carcinoma & 1 & 0.6 \\
Familial Mediterranean fever & 1 & 0.6 \\
Idiopathic thrombocytopenic purpura & 1 & 0.6 \\
Previous ileal resection & 1 & 0.6 \\
Total & 62 & 38.3 \\
\hline
\end{tabular}

increased respiration. The patient was feeling an urge to breathe deeply, an "air hunger", almost involuntary with fever $39.5 \mathrm{C}$ and tachycardia 160/minutes. Though her two previous tests for SARS-COV-2 was negative, the third test revealed COVID-19 in diabetic ketoacidosis. Laboratory tests, typical for COVID-19: White Blood Cells: 11.7 (3.9-10.2), neutrophils: 10.42 (normal levels: 1.5-7.7), lymphocytes: 0.48 (normal levels: 1.1-4.5). Ferritin: 406 (normal levels: 10-291), procalcitonin: 0.71 (normal levels: $<0.16$ ) and $C$ reactive protein: 0.247 (normal levels: 0-0.005) were the reasons for the repetition of the tests. Another woman with diabetes mellitus had deteriorating respiratory insufficiency due to pandemic pneumonia that required oxygen supplementation with high flow nasal cannula at 30 weeks of gestation. The only pregnant woman with critical disease with no known co-morbidity developed inability to arouse. Respiratory insufficiency, acidosis, cardiac involvement of COVID-19 detoriated her status. She died in the intensive care unit 15 days after admission and in the 11th day of ICU despite thromboprophylaxis [20], antiviral and antibiotic therapy (Table 2).

First-trimester pregnancy loss appeared to be high (Table 2). There were three mid-trimester perinatal deaths. Three women at 28,29 and 33 weeks, required intensive care unit admission due to $50 \%$ lung involvement and underwent iatrogenic preterm delivery. Pregnancy outcome was available in 83 women $(51.2 \%)$ and 67 women (41.4\%) delivered during the study period (Table 2). None of the neonates delivered 


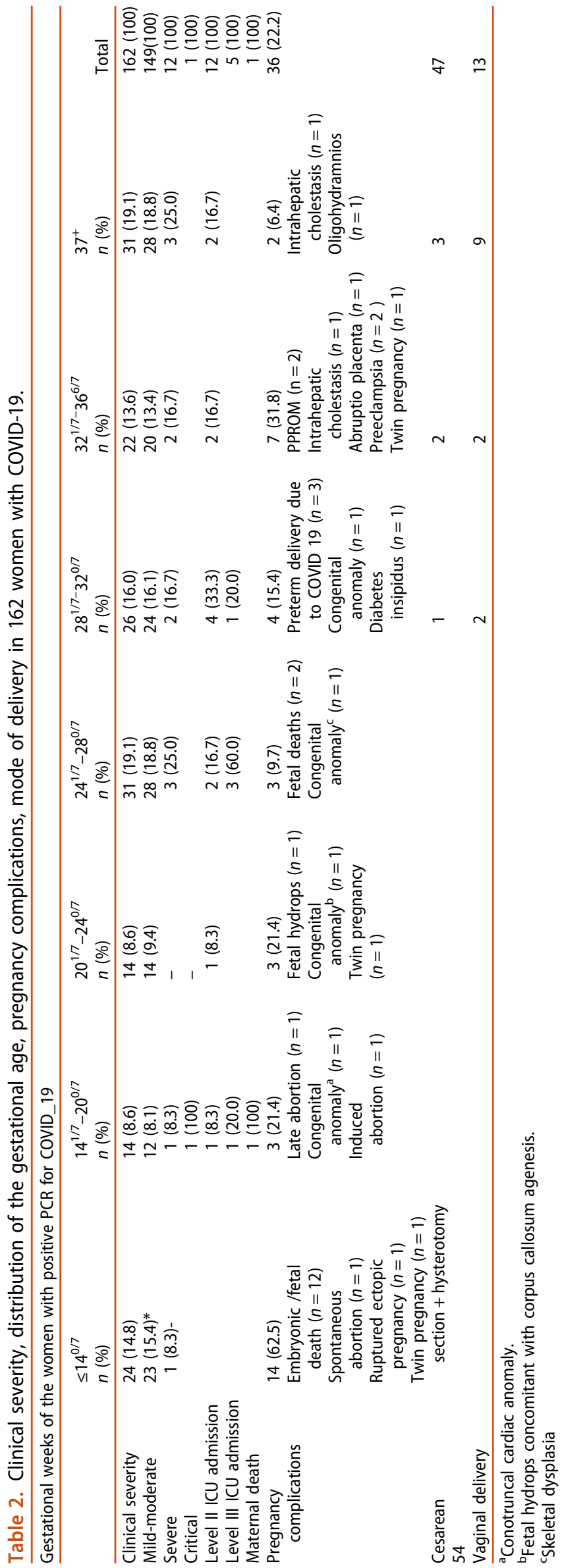

at City hospital was positive for SARS-CoV-2 and breastmilk specimen samples were also negative for the virus. In comparison of the births, CS rates (Table 3), ultrasound examinations, surgical procedures (carman aspiration, dilatation and curettage, cerclage, placental removal, Bartholin cyst extirpation), fetal invasive procedures, number of the fetal structural abnormalities (Table 4), intensive care unit admission (Table 5) during pandemic with those in the preceding three months revealed continuing work and high CS rates despite lock-down. Obstetricians/perinatologists/ assistant doctors delivered women. ICU admission due to preeclampsia increased in lockdown (Table 5). While providing health care, $25 \mathrm{HCW}$ developed SARSCOV-2 positivity. Of those 22 were working in the perinatology department or very close contact (Figure 2). Frontline HCW: nurses/midwives, doctors and health technicians, directly providing services for the enhancement of health were affected with a ratio of $84 \%$.

\section{Discussion}

In the Social Security Law of Turkey, maternity as a risk is covered both by the universal health insurance for health care services and by a specific insurance brand under Act no 5510 [21]. The risk of maternity is regulated in a manner very similar to insurance for sickness, allowing the chance for all pregnant women to take antenatal care and free birth service from hospitals of the government and universities. In this study, we presented the statistics of a nonstop perinatology clinic during COVID-19 pandemic. Clinical features of 162 pregnant women with positive RT-PCR for SARS-COV-2 and $5.4 \%$ rate of horizontal transmission in the exposed HCW were documented. Illness severity was mild to moderate in $90 \%$ of the pregnant women whereas there were severe $(7.4 \%)$ and critical $(0.6 \%)$ cases. One maternal death occurred. The horizontal transmission was asymptomatic (32\%), mildmoderate (60\%) and severe (8\%) in the HCW.

United Kingdom Obstetric Surveillance System (UKOSS) reported the complete data on 427 pregnant women with COVID-19: 38 women (9\%) required level-3 critical care; four women $(<1 \%)$ received extracorporeal membrane oxygenation and five maternal deaths, suggesting a SARS-CoV-2-associated maternal mortality rate of 5.6 (95\% Cl 1.8-13.1) per 100,000 maternities [22]. In a report from Iran, among 9 pregnant women with severe COVID-19 disease, at second or third trimesters, maternal death occurred in 7 of 9 women. 1 of 9 remained critically ill and ventilator dependent, and 1 of 
Table 3. Distribution of the births from 2019 December to August 2020.

\begin{tabular}{|c|c|c|c|c|c|c|c|c|}
\hline \multirow{3}{*}{$\begin{array}{l}\text { Year } \\
\text { Month } \\
\text { Laborward }\end{array}$} & \multirow{3}{*}{$\begin{array}{c}2019 \\
12 \\
n(\%)\end{array}$} & \multicolumn{7}{|c|}{2020} \\
\hline & & 1 & 2 & 3 & 4 & 5 & 6 & 7 \\
\hline & & $n(\%)$ & $n(\%)$ & $n(\%)$ & $n(\%)$ & $n(\%)$ & $n(\%)$ & $n(\%)$ \\
\hline \multicolumn{9}{|l|}{ Low risk women } \\
\hline Vaginal births & $485(71.5)$ & $507(68.8)$ & $441(59.3)$ & $476(59.6)$ & $405(58.4)$ & $323(50.0)$ & 591.(62.0) & $657(57.4)$ \\
\hline Total CS & $310(38.5)$ & $326(31.2)$ & $303(40.7)$ & $323(40.4)$ & $289(41.6)$ & $323(50.0)$ & $362(38.0)$ & $487(42.6)$ \\
\hline Primary CS & 121(15.6) & $120(14.4)$ & $101(13.6)$ & $114(14.3)$ & $113(16.3)$ & $126(19.5)$ & $161(16.9)$ & 213 (18.6) \\
\hline Total livebirths & $804(100)$ & $833(100)$ & $744(100)$ & $799(100)$ & $694(100)$ & $646(100)$ & $953(100)$ & $1144(100)$ \\
\hline \multicolumn{9}{|l|}{ Perinatology } \\
\hline \multicolumn{9}{|l|}{ High-risk women } \\
\hline Vaginal births & $86(28.4)$ & $74(24.8)$ & $71(28.6)$ & $73(26.4)$ & $52(29.7)$ & $69(27.0)$ & $100(31.2)$ & $100(31.9)$ \\
\hline Total CS & 217 (71.6) & $224(75.2)$ & 177 (71.4) & $201(73.6)$ & $132(71.3)$ & $154(63.0)$ & $221(68.8)$ & $213(68.1)$ \\
\hline Primary CS & $113(37.3)$ & $125(43.1)$ & $92(37.1)$ & $104(37.9)$ & $70(37.8)$ & $97(43.5)$ & $130(40.50)$ & $99(31.6)$ \\
\hline Multiple pregnancies & $17^{*}(5.6)$ & $18^{*}(6.0)$ & $11^{*}(4.4)$ & $28 *(10.2)$ & $14^{*}(7.7)$ & $25(11.2)$ & $25(7.8)$ & $20(6.3)$ \\
\hline Perinatal deaths & $13(4.2)$ & $19(6.4)$ & $15(6.0)$ & $13(4.7)$ & $8(4.3)$ & $19(8.5)$ & $16(5.0)$ & $16(5.1)$ \\
\hline Number of births & 303 & 298 & 248 & 274 & 185 & 223 & 321 & 313 \\
\hline
\end{tabular}

*All twins but 1 triplet pregnancy. All multiples were delivered by CS.

9 recovered after a prolonged hospitalization [23]. The MERS [30] and 10.5\% in SARS [8] and increased rates of Table 4. Number of the women evaluated at perinatology clinic and the tests and procedures performed between 1 December 2019 and 1 August 2020.

\begin{tabular}{|c|c|c|c|c|c|c|c|c|}
\hline & December 2019 & January 2020 & February 2020 & March 2020 & April 2020 & May 2020 & June 2020 & 2020 July \\
\hline Perinatology outpatient examination & 2883 & 3268 & 3131 & 2518 & 1332 & 1728 & 2392 & 2233 \\
\hline Perinatology admission & 542 & 520 & 447 & 449 & 280 & 359 & 488 & 445 \\
\hline Transabdominal ultrasound & 2883 & 3268 & 3131 & 2518 & 1332 & 1728 & 2392 & 2233 \\
\hline Transvaginal ultrasound & 262 & 355 & 271 & 176 & 87 & 143 & 192 & 190 \\
\hline Doppler & 1158 & 1100 & 898 & 709 & 482 & 711 & 887 & 810 \\
\hline Fetal cardiotocography & 2987 & 3315 & 3085 & 3084 & 1980 & 2373 & 4066 & 3379 \\
\hline Pregnancy termination ${ }^{b}$ & 15 & 16 & 22 & $18^{\mathrm{a}}$ & 5 & 15 & 16 & 15 \\
\hline Surgical procedures & 98 & 223 & 219 & 159 & 101 & 143 & 183 & 98 \\
\hline Fetal death & 35 & 30 & 35 & 29 & 23 & 22 & 35 & 30 \\
\hline Abortion & 4 & $9^{a}$ & $4^{a}$ & $4^{a}$ & 5 & 3 & 4 & $9^{a}$ \\
\hline Amniocentesis & 26 & 32 & 43 & 25 & 17 & 12 & 39 & 25 \\
\hline CVS & 3 & 10 & 10 & 8 & 5 & 5 & 4 & 7 \\
\hline Fetal blood sampling & 1 & - & 3 & 1 & 1 & 1 & 2 & 1 \\
\hline Fetal transfusion & & & & 3 & 1 & 1 & 1 & \\
\hline Fetoside + multifetal pregnancy reduction & & 3 & 3 & 2 & 4 & 2 & 3 & 7 \\
\hline Amnioreduction & & 3 & & & & & 1 & 1 \\
\hline Vesiculocentesis & & & & & & & & 2 \\
\hline Fetal stent insertion & & & & & 1 & & & 1 \\
\hline Laser treatment for TTS & & 1 & & & & & & \\
\hline
\end{tabular}

${ }^{\mathrm{a} C o-t w i n .}$

${ }^{\mathrm{b}}$ Gestational age $>10$ weeks.

importance of the study from Iran is the advice that caution against complacency and guide restraint in rushing estimates of relative or attributable risk with pregnancy; i.e. at about $1 \%$. The dynamics of the immune alterations during pregnancy, lung physiology [24] and evidence of increased generation of thrombin and a pro-thrombotic state as well increased intravascular inflammation that is exaggerated in the context of infection [20] may make pregnant women more vulnerable to a more severe course of the COVID-19.

The more severe disease courses in pregnant women during influenza [25-27], influenza A virus subtype H1N1 [28], SARS [29] and the Middle East Respiratory Syndrome (MERS) pandemics [30] have raised important concerns regarding the course of COVID-19 in obstetrical patients as high case fatality rates: $34.4 \%$ in the intensive care unit admissions were reported. However, though there is one death just before the completion of the paper, the rates may subject to change in the evolving course of the pandemic. Data from the UKOSS depicted that most women were hospitalized in the third trimester or peripartum $(n=342,81 \%)$ with the median gestational age at hospital admission was 34 completed weeks (interquartile range [IQR] 29-38) [21], while our data show a homogeneous distribution in the first 13 weeks' gestation and at 20, 26, 30, 34, 36, 38 and 40 weeks' gestation depicting the continuation of regular antenatal care. Of those, while first-trimester loss appeared to be more frequent, fetal deaths occurred in four and iatrogenic preterm delivery in three women with severe disease. Of those who did give birth during the data collection period, the CS rate 
Table 5.

\begin{tabular}{|c|c|c|c|c|}
\hline Number of the women & $\begin{array}{l}\text { Before pandemic } \\
n\end{array}$ & $\begin{array}{c}\text { Pandemic } \\
n\end{array}$ & $\begin{array}{c}\text { Total } \\
n\end{array}$ & $p$ \\
\hline Pre-eclampsia & 62 & 84 & 146 & .004 \\
\hline HELLP & 5 & 5 & 10 & $>.05$ \\
\hline Eclampsia & 1 & 2 & 3 & $>.05$ \\
\hline Epilepsy $^{\mathrm{a}}$ & 10 & 7 & 17 & $>.05$ \\
\hline Postpartum hemorrhage & 14 & 10 & 25 & $>.05$ \\
\hline Placenta previa + accreta & 5 & 8 & 13 & $>.05$ \\
\hline Renal failure & - & 3 & 3 & $>.05$ \\
\hline Abruptio placenta & 3 & 2 & 5 & $>.05$ \\
\hline Cardiac failure & 2 & 3 & 5 & $>.05$ \\
\hline Heart disease & 2 & 5 & 7 & $>.05$ \\
\hline ITP & 2 & 1 & 3 & $>.05$ \\
\hline Diabetic ketoacidosis & 1 & 1 & 2 & $>.05$ \\
\hline Acute abdomen & 1 & 1 & 2 & $>.05$ \\
\hline Anesthesia related & 2 & - & 2 & $>.05$ \\
\hline ERCP + stent replacement & 1 & - & 1 & $>.05$ \\
\hline Subcutaneous emphysema & 1 & - & 1 & $>.05$ \\
\hline End stage breast carcinoma & 1 & - & 1 & $>.05$ \\
\hline Asthma & 1 & - & 1 & $>.05$ \\
\hline Paraacetamol intoxication & 1 & - & 1 & $>.05$ \\
\hline Septic abortion & 1 & - & 1 & $>.05$ \\
\hline Pulmonary embolism & 1 & - & 1 & $>.05$ \\
\hline Portal hypertension & 1 & - & 1 & $>.05$ \\
\hline Suicide attempt & 1 & - & 1 & $>.05$ \\
\hline Motor vehicle accident & 1 & - & 1 & $>.05$ \\
\hline Deep vein thrombosis & 1 & - & 1 & $>.05$ \\
\hline Hemolytic uremic syndrome & 1 & - & 1 & $>.05$ \\
\hline Maternal deaths & $2^{b}$ & - & 2 & $>.05$ \\
\hline Hemofiltration & 2 & - & 2 & $>.05$ \\
\hline Plasmapheresis & 2 & - & 2 & $>.05$ \\
\hline Total & 128 & 129 & 257 & \\
\hline
\end{tabular}

ERCP: endoscopic retrograde cholangio-pancreatography.

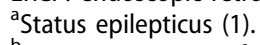

${ }^{b}$ One woman was referred after intracranial hemorrhage. Another woman developed massive mesenteric vein thrombosis in the 5th day after CS despite thromboprophylaxis.

was high; approximately half of these were due to repeat CS.

As it is neither possible to eradicate COVID-19 by any measures currently, nor reduce the number of deliveries, emergent complications, fetal abnormalities and timely diagnostic invasive procedures, $\mathrm{HCW}$ are at serious risk for the horizontal transmission. Asymptomatic pregnant women rate of $\% 1.5$ with positive PCR for SARS-COV-2 at the beginning of the pandemic [6] may no longer be valid due to the increase in the COVID-19 cases and the tests performed. Increased frequency of positive test results in asymptomatic pregnant women for COVID-19, up to $13.5 \%$ reported by Sutton et al. [8], and similarly $13.3 \%$ by London et al. [31]. The risk is not decreasing, in fact, maybe increasing as although many countries have made some progress, globally the pandemic appears to be speeding up within the process of "normalization and appears to be "not even close to being over" in Turkey as in most of the world. In our report, "Exposed" group in the SuEIR model are the HCW that have already been infected and have not been tested, which have been also capable of infecting the "Susceptible" group. Moreover, some of the HCW in the "Exposed" group were tested and transferred to the "Infectious" group (which were reported to be PCR positive), while the rest of them who recovered, transitted to the so-called "Unreported Recovered" group. In the current chaotic screening policy for both the pregnant women and the HCW for COVID-19, potentially valuable information is likely to be obtained by perinatologists/obstetricians that are in the frontline during birthing processes, antenatal care, ultrasonographic and Doppler evaluation, fetal invasive procedures and vaginal examinations both to improve the understanding of the pathophysiology of COVID-19 infection, vertical and horizontal transmission.

Our study may provide information on the horizontal transmission of COVID-19 during perinatal care in City Hospital: the optimum use of PPE by the HCW and the pregnant women to combat the threat of COVID-19. However, a physical distancing of at least 1 $\mathrm{m}$, strongly associated with protection could neither be achieved during births nor in procedures involving fetal diagnosis and therapy while a protective shield that functions as a physical barrier for vaginal delivery has been in use [32]. A horizontal transmission rate of $5.4 \%$ in the frontline $\mathrm{HCW}$ is open to debate according to SuEIR model. Our findings may be generalizable to neither our hospital in the near future nor other centers or regions. The horizontal transmission from HCW 


\section{PERINATOLOGY}

3 Perinatology consultants

4 Subspecialty specialists

15 Perinatology fellows

150 Obstetrics/gynecology assistants

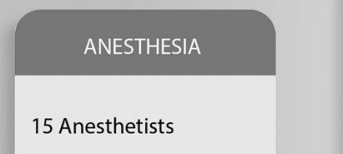

27 Anesthesia technicians

NEONATOLOGY

16 Neonatologists

OTHER HEALTHCAPE

WORKERS

178 Midwives/Nurses
FRONTLINE MEDICAL STAFF

COVID-19 HORIZONTAL TRANSMISSION

7 Doctors

Obstetrics/gynecology assistants (4)

Anesthesia assistant (1)

Radiologist (1)

Workplace doctor (1)

11 Midwives/nurses

3 Health technicians

1 Pharmacist

2 Data processing employees

1 Security employee

25 Healthcare workers in total

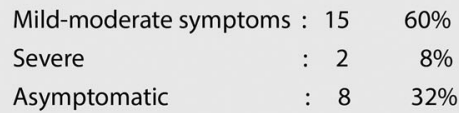

Asymptomatic $\quad: \quad 8 \quad 32 \%$

Figure 2. Horizontal transmission and severity of COVID-19 in Perinatology unit health-care workers.

to HCW should also be considered. We believe a strategy of universal testing for pregnant women with a previous CS, that is being undertaken since June 2020 may help detect asymptomatic pregnant women as repeat CS contributed to half of the CSs in Turkey [2]. Although asymptomatic [33] and pre-symptomatic [34] carriers can infect others, their period of contagiousness is unknown $[35,36]$. In conclusion: Due to the continuing obstetric care and high rate of emergency conditions during pandemic, perinatology/obstetric HCWs are at extremely increased risk. Where universal screening for all women may not be performed due to lack of resources and technical reasons, clinical triage, standard precautions and PPE together with the concept, "all have COVID-19" may help to alleviate the horizontal transmission. Implementing appropriate strategies during COVID-19 requires both political commitment and public engagement in combination with investing in maternal and perinatal health [37] more than ever.

\section{Glossary}

Kalman Filter (also called as linear quadratic estimation): an algorithm that uses a series of measurements observed over time, containing statistical noise and other inaccuracies, and produces estimates of unknown variables that tend to be more accurate than those based on a single measurement alone, by estimating a joint probability distribution over the variables for each timeframe. The filter is named after Rudolf E. Kalman, one of the primary developers of its theory.

Unscented Kalman filter: Extensions and generalizations of Kalman filter that have been developed which work on nonlinear systems.

\section{Disclosure statement}

No potential conflict of interest was reported by the author(s).

\section{ORCID}

Ozlem Moraloglu Tekin (D) http://orcid.org/0000-0001-

8167-3837

Dilek Sahin (D) http://orcid.org/0000-0001-8567-9048

Filiz Halici Ozturk (D) http://orcid.org/0000-0003-4049-4140

\section{References}

[1] Turkish Statistical Institute. 2020; [cited 2020 Feb 23]. Available from: tuik.gov.tr. 02-04.

[2] Yapar Eyi EG, Mollamahmutoglu L. An analysis of high cesarean section rates in Turkey by Robson classification. J Matern Fetal Neonatal Med. 2019:1-11. Published online 01 Oct 2019. DOI:10.1080/14767058.2019.1670806 
[3] Zou D, Wang L, Xu P, et al. Epidemic model guided machine learning for COVID-19 Forecasts in the United States. Available from: https://covid19.uclaml.org/.

[4] Onat A. A novel and computationally efficient joint unscented kalman filtering scheme for parameter estimation of a class of nonlinear systems. IEEE Access. 2019;7:31634-31655.

[5] Breslin N, Baptiste C, Gyamfi-Bannerman C, et al. Coronavirus disease 2019 infection among asymptomatic and symptomatic pregnant women: two weeks of confirmed presentations to an affiliated pair of New York City hospitals. Am J Obstet Gynecol Mfm. 2020;2(2):100118.

[6] Tanacan A, Erol SA, Turgay B, et al. The rate of SARSCoV-2 positivity in asymptomatic pregnant women admitted to hospital for delivery: experience of a pandemic center in Turkey. Eur J Obstet Gynecol Reprod Biol. 2020; 253:11-34.

[7] Khalil A, Hill R, Ladhani S, et al. SARS-CoV-2 in pregnancy: symptomatic pregnant women are only the tip of the iceberg. Am J Obstet Gynecol. 2020;2;223.

[8] Sutton D, Fuchs $K, D^{\prime} A l t o n ~ M$, et al. Universal screening for SARS-CoV-2 in women admitted for delivery. N Engl J Med. 2020;382(22):2163-2164.

[9] LaCourse SM, Kachikis A, Blain $M$, et al. Low prevalence of SARS-CoV-2 among pregnant and postpartum patients with universal screening in Seattle, Washington. Clin Infect Dis. 2020. DOI:10.1093/cid/ ciaa675. Epub ahead of print.

[10] Dória M, Peixinho C, Laranjo M, et al. Covid-19 during pregnancy: a case series from an universally tested population from the north of Portugal. Eur J Obstet Gynecol Reprod Biol. 2020;250:261-262.

[11] Chu DK, Akl EA, Duda S, et al. Holger J Schünemann, on behalf of the COVID-19 Systematic Urgent Review Group Effort (SURGE) study authors. Physical distancing, face masks, and eye protection to prevent person-to-person transmission of SARS-CoV-2 and COVID-19: a systematic review and meta-analysis. Lancet. 2020;395(10242):1973-1987.

[12] Turkish Ministry of Health. General directorate of public health, COVID-19 (SARS-CoV-2 infection) guideline. Scientific Committee Report; 2020; [cited 2020 June 19]. Available from: https://covid19.bilgi.saglik.gov.tr/ depo/rehberler/COVID19-Rehberi

[13] Bartoszko JJ, Farooqi MAM, Alhazzani W, et al. Medical masks vs $\mathrm{N} 95$ respirators for preventing COVID-19 in health-care workers: a systematic review and meta-analysis of randomized trials. Influenza Other Respir Viruses. 2020;14(4):365-373.

[14] Capanna F, Haydar A, McCarey C, et al. Preparing an obstetric unit in the heart of the epidemic strike of COVID-19: quick reorganization tips. J Matern Fetal Neonatal Med. 2020;12:1-7. DOI:10.1080/14767058. 2020.1749258.

[15] Lippi G, Simundic A, Plebani M. Potential preanalytical and analytical vulnerabilities in the laboratory diagnosis of coronavirus disease 2019 (COVID-19). Clin Chem Lab Med. 2020;58(7):1070-1076.

[16] Tahamtan A, Ardebili A. Real-time RT-PCR in COVID19 detection: issues affecting the results. Expert Rev Mol Diagn. 2020;20(5):453-454.
[17] Guan W-j, Ni Z-y, Hu Y, et al. Clinical characteristics of Coronavirus Disease 2019 in China. N Engl J Med. 2020;382(18):1708-1720.

[18] Wang D, Hu B, Hu C, et al. Clinical characteristics of 138 hospitalized patients with 2019 novel Coronavirus-Infected Pneumonia in Wuhan, China. JAMA. 2020;323(11):1061-1069.

[19] Minagar A, Weiner WJ. Adolf Kussmaul and his respiratory sign. J Med Biogr. 2001;9(3):181-183.

[20] Di Renzo GC, Giardina I. Coronavirus disease 2019 in pregnancy: consider thromboembolic disorders and thromboprophylaxis. Am J Obstet Gynecol. 2020; 223(1):135

[21] 5510 Social Insurance and Universal Health Insurance Law II. The Republic of Turkey Ministry of Labor and Social Security. 35th Detailed Country Report Prepared by The Government of the Republic of Turkey in Accordance with the Article 74 of the European Code of Social Security (Reference Period: 01.07.2011-30.06.2016)

[22] Knight M, Bunch K, Vousden N, et al. UK Obstetric Surveillance System SARS-CoV-2 Infection in Pregnancy Collaborative Group. Characteristics and outcomes of pregnant women admitted to hospital with confirmed SARS-CoV-2 infection in UK: national population based cohort study. BMJ. 2020;369:m2107.

[23] Hantoushzadeh S, Shamshirsaz AA, Aleyasin A, et al. Maternal death due to COVID-19. Am J Obstet Gynecol. 2020;223(1):109.e1-109.e16.

[24] Forestieri S, Marcialis MA, Migliore L, et al. Relationship between pregnancy and coronavirus: what we know. J Matern Fetal Neonatal Med. 2020;4: 1-12. DOI:10.1080/14767058.2020.1771692.

[25] Callaghan WM, Creanga AA, Jamieson DJ. Pregnancyrelated mortality resulting from influenza in the United States during the 2009-2010 Pandemic. Obstet Gynecol. 2015;126(3):486-490.

[26] Harris JW. Influenza occurring in pregnant women: a statistical study of thirteen hundred and fifty cases. JAMA. 1919;72(14):978-980.

[27] Freeman DW, Barno A. Deaths from Asian influenza associated with pregnancy. Am J Obstet Gynecol. 1959;78:1172-1175.

[28] Jamieson DJ, Honein MA, Rasmussen SA, et al. H1N1 2009 influenza virus infection during pregnancy in the USA. Lancet. 2009;374(9688):451-458.

[29] Wong SF, Chow KM, Leung TN, et al. Pregnancy and perinatal outcomes of women with severe acute respiratory syndrome. Am J Obstet Gynecol. 2004; 191(1):292-297.

[30] Alfaraj SH, Al-Tawfiq JA, Memish ZA. Middle East Respiratory Syndrome Coronavirus (MERS-CoV) infection during pregnancy: report of two cases \& review of the literature. J Microbiol Immunol Infect. 2019; 52(3):501-503.

[31] London V, McLaren R, Jr, Atallah F, et al. The relationship between status at presentation and outcomes among pregnant women with COVID-19. Am J Perinatol. 2020;37(10):991-994.

[32] Sahin D, Erol A, Tanacan A, et al. Protective equipment to use in the vaginal delivery of the pregnant woman with suspected or diagnosed coronavirus 
disease 2019: delivery table shield. Am J Obstet Gynecol. 2020; 223(4):599-601.

[33] Deprest J, Choolani M, Chervenak F, et al. Fetal diagnosis and therapy during the COVID-19 Pandemic: guidance on behalf of the International Fetal Medicine and Surgery Society. Fetal Diagn Ther. 2020; 47(9):689-698.

[34] Rothe C, Schunk M, Sothmann P, et al. Transmission of 2019-nCoV Infection from an asymptomatic contact in Germany. N Engl J Med. 2020;382(10): 970-961.
[35] Wei WE, Li Z, Chiew CJ, et al. Presymptomatic transmission of SARS-CoV-2 - Singapore, January 23-March 16, 2020. Morb Mortal Wkly Rep. 2020; 69(14):411-415.

[36] Wang W, Xu Y, Gao R, et al. Detection of SARS-CoV-2 in different types of clinical specimens. JAMA. 2020; 323(18):1843-1844.

[37] Jacob CM, Briana DD, Di Renzo GC, et al. Building resilient societies after COVID-19: the case for investing in maternal, neonatal, and child health. Lancet Public Health. 2020;5(11):e624-e627. 